\title{
Electrocardiographic and Phonocardiographic Studies in Hypertension
}

\author{
Part II. Phonocardiographic Study with Special Reference to \\ the Atrial Sound and "Q-1" Interval
}

Tsuguya SAKAMOTO, M.D., Goro KAITO, M.D., and Hideo UEDA, M.D.*

Phonocardiographic study was made in order to display the similarity in the left atrial overloading between hypertension and mitral stenosis which is expressed by the P-wave changes as described in the preceeding paper. It was concluded that the accentuation of the atrial sound and the prolongation of the "Q-1" interval, combined with the $\mathrm{P}$-wave changes, were the reflection of the left atrial overloading, therefore, these findings might be one of the objective signs indicating the impending left heart failure in hypertension.

I $\mathrm{N}$ the previous paper, ${ }^{1)}$ a certain similarity in the $\mathrm{P}$-wave of the electrocardiogram between the cases with hypertension and mitral stenosis was discussed. It was concluded then that this similarity was due to the overloading of the left atrium which appeared in accordance with elevated diastolic blood pressure in hypertension. On the other hand, such a similarity may be also present in the mechanical phenomena in both conditions. In the present study, an attempt was made to evaluate this similarity by means of the phonocardiogram. In addition, the correlation between the electrocardiographic and phonocardiographic findings was also studied.

\section{Methods and Materials}

A group of patients was selected from the previous study" at random, which consisted of 50 normal, 150 hypertensive and 41 cases with pure mitral stenosis. The follow-up cases and 18 hypertensive cases subjected to cold pressor test were the same in the preceeding report. The cases with widening of QRS complex were excluded.

Using a multi-filter system phonocardiograph, a modification of a system made by Yoshimura's, a phonocardiogram (PCG) was recorded in these patients. Frequency-response characteristics of the amplifier with 5 filters of this phonocardiograph is shown in Fig. 1. Records were made by moving-coil electromagnetic oscillograph, which had a flat response up to $1,000 \mathrm{cps}$. With this device, the heart sounds and murmurs with wide frequency range were satisfactorily recorded in any PCG with a suitable filter. Paper speed was $80 \mathrm{~mm}$. and $120 \mathrm{~mm}$. per sec. and these speeds facilitated for the measurement up to $0.01 \mathrm{sec}$. The dynamic microphone with a chest piece of $35 \mathrm{~mm}$. in diameter was used and it gave a Tokyo.

From the Second Department of Internal Medicine, Faculty of Medicine, University of Tokyo,

* Professor of Internal Medicine. 


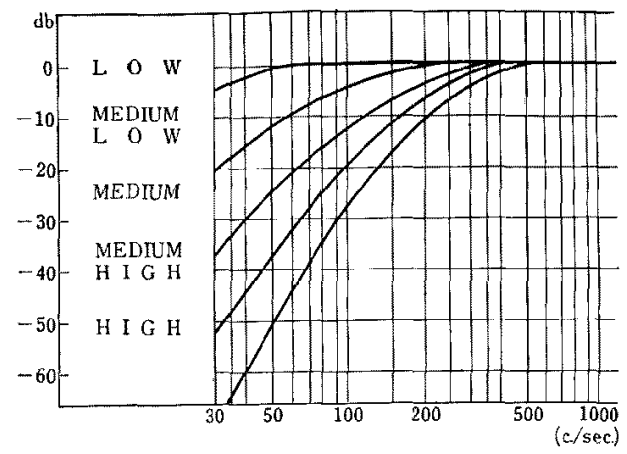

Fig. 1. Frequency-response characteristics of the amplifier. The degree of the attenuation of $50 \mathrm{cps}$ for $500 \mathrm{cps}$ is $0 \mathrm{db}$ in filter 1 (Low), $12.5 \mathrm{db}$ in filter 2 (Medium Low), $25 \mathrm{db}$ in fiter 3 (Medium), $37.5 \mathrm{db}$ in filter 4 (Medium High) and $50 \mathrm{db}$ in filter 5 (High). In addition to these five filters, this amplifier is equipped with a filter, which has $62.5 \mathrm{db}$ of attenuation, and it is sometimes used for recording of the particularly high-pitched murmurs. The degree of the same attenuation due to the microphone is $15 \mathrm{db}$ in each $\mathrm{PCG}$.

constant pressure of $200 \mathrm{Gm}$. to the chest wall. In addition, this phonocardiograph is equipped with a calibration device $(5 \mathrm{mV}$., $50 \mathrm{cps}$.; alternate current) at the input stage of the amplifier. However, because of many complicating problems on the calibration of the heart sounds, this method was individualized in each subject and was not used for the purpose of comparison in the different cases.

Phonocardiographic recording was taken simultaneously with electrocardiogram (lead II), and when the Q-wave was absent or questionable in this lead, the beginning of the QRS complex was determined by the simultaneous recording of multiple leads (usually 3 standard leads). Records were made with the patients in a supine position at the end of expiration with the glottis open, and at the various auscultatory areas.

Atrial sound: The loudness of the atrial sound was classified according to the difference of $\mathbf{P G G}$, up to which the sound was recognized. For example, grade I was decided when the sound appeared in only Low PCG, and when it was recognized in every PCG, the grade $V$ was decided. Thus, "the grade of accentuation " of atrial sound was classified into 5 groups (from I to V). This method was also used for the comparison of atrial sound in the follow-up cases.

"Q-1" interval: "Q-1" interval was measured in the apical Medium PCG by the interval between the $Q$-wave and the beginning of the first major deflection of the first sound. A special attention was given to determine and differentiate the beginning of the first sound from the ejection component. In cases which showed the diminished first sound and the accentuated ejection sound, this attention was particularly needed. In these instances, it was necessary to compare the Medium PCG with the one of Medium Low and/or Low, which showed a clear cut first sound. Additionally, 50 cases of normal younger persons were used for the comparison study of the "Q-1" interval.

During cold pressor test, the microphone on the chest wall was firmly fixed, and the gain of the amplifier was maintained constant in order to compare the recordings accurately. The amplitude of the atrial sound was measured on the average of at least 4 in the apical Low PCG before and after the test. No significant change was 
seen in the heart rate during this test. T-test was performed for the significance of the analysis.

\section{Results}

1) Atrial sound:

In a total of 50 normal cases studied, 3 appeared to show the atrial sound up to Medium PGG (grade III). Remaining 47 cases showed

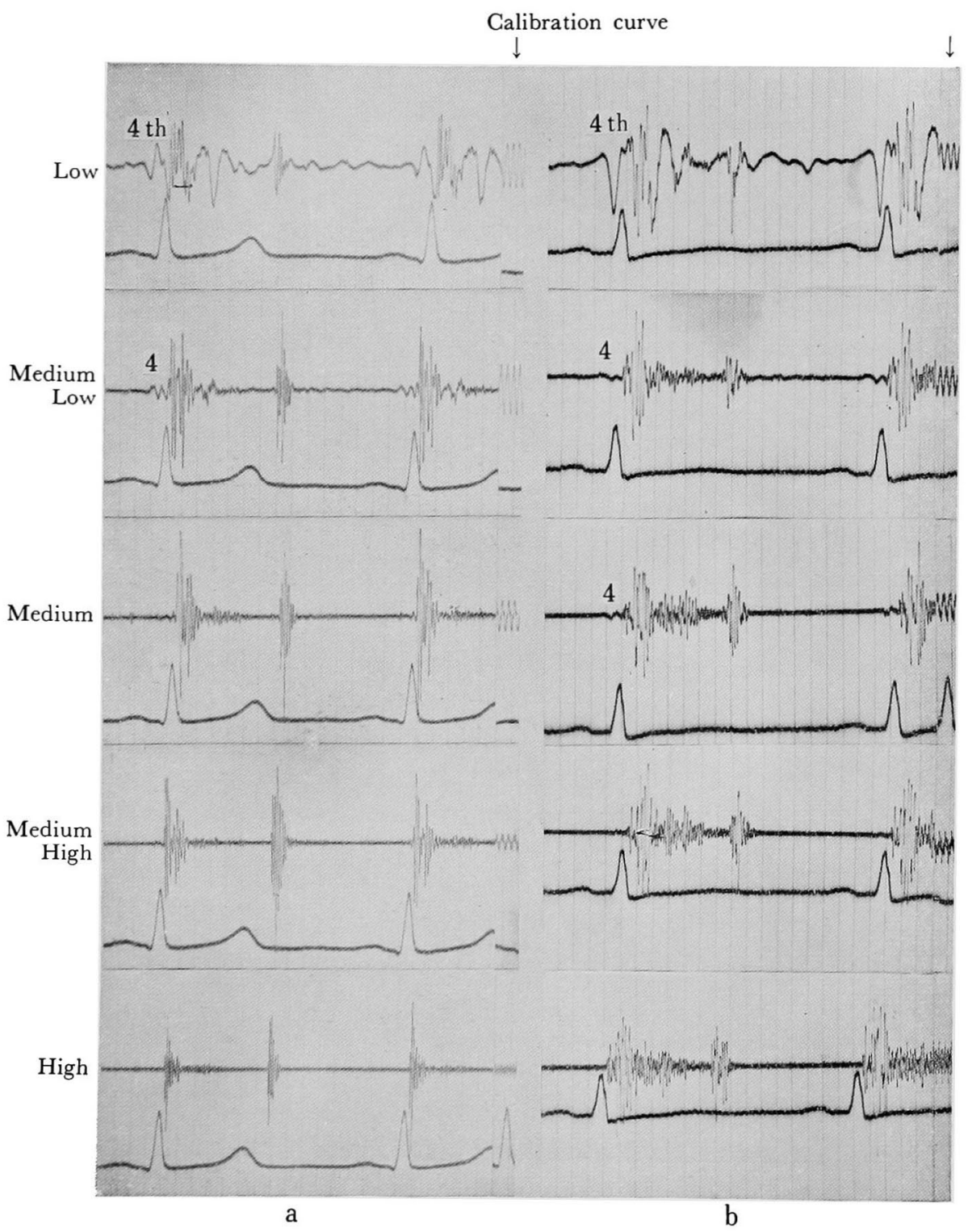

Fig. 2. Phonocardiogram in the cases with mild hypertension (Apex). The atrial sound in the case a appears up to Medium Low PCG (grade of accentuation is II). This grade in the case $\mathbf{b}$ is III. 
definite atrial sound in Low PCG (grade I) or up to Medium Low PCG (grade II). On the other hand, the atrial sound in hypertensive became louder and was seen in even the higher characteristic PCG (Figs. 2 and 3). This accentuation of the atrial sound had some correlaton with the diastolic blood pressure as seen in Fig. 4. In 15 hypertensive cases with congestive heart failure, 13 belonged to grade IV or more, and the atrial sound in these cases was audible in all (Fig. 3). The accentuated atrial sound beyond the grade III was usually audible. The maximal intensity of this sound was

Calibration curve

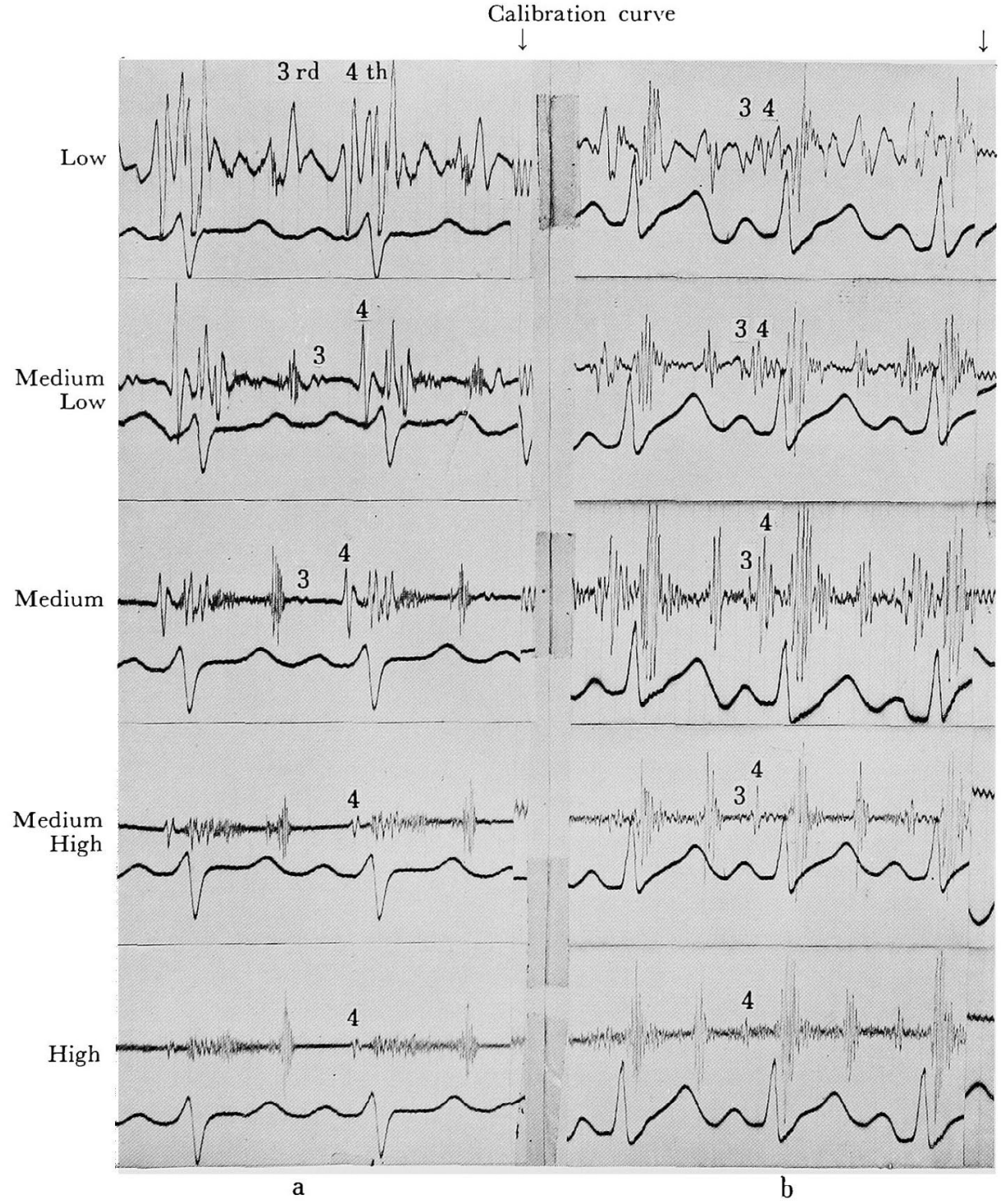

Fig. 3. Phonocardiogram in the cases with severe hypertension (Apex). Quadruple rhythm was heard in both cases. A loud atrial sound appears up to High PCG (grade V). The third sound in hypertension usually appears in the cases with marked decompensation. 


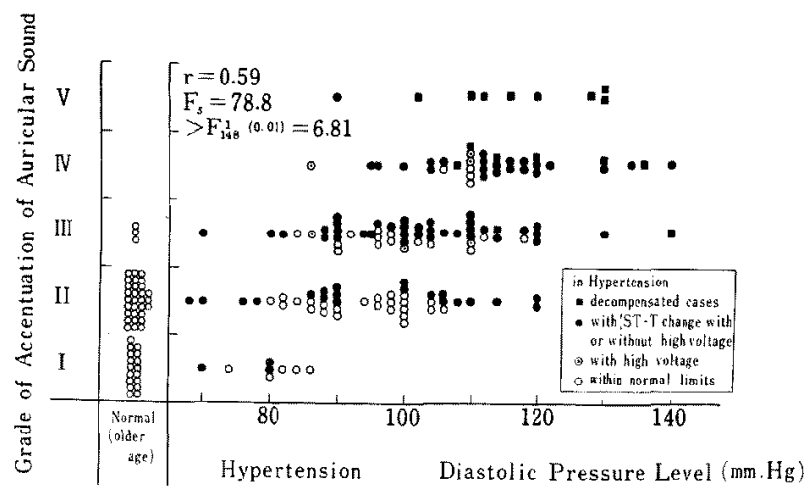

Fig. 4. Rclationship between the grade of accentuation of the atrial sound and the diastolic blood pressure level in hypertension. (In succeeding figures, the same symbols are used as to hypertensive cases. The correlation coefficients in this study are calculated on the assumption that the various grades are successive value).

2) "Q-1" interval: (Table I)

Table I. Average Value of "Q-1" Interval

\begin{tabular}{|c|c|c|c|c|}
\hline & & No. of cases & \multicolumn{2}{|c|}{ "Q-1" interval (sec.) } \\
\hline \multicolumn{2}{|c|}{ Normal adults } & 50 & .051 & \multirow{2}{*}{$\begin{array}{r}.050 \\
\pm .006\end{array}$} \\
\hline \multicolumn{2}{|c|}{ Normal control (younger age) } & 50 & .050 & \\
\hline \multirow{6}{*}{ 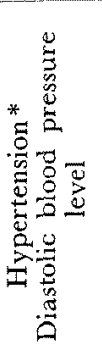 } & $\sim 89 \mathrm{~mm} . \mathrm{Hg}$ & 31 & .055 & \multirow{6}{*}{$\begin{array}{r}.064 \\
\pm .010\end{array}$} \\
\hline & $90 \sim 99$ & 30 & .060 & \\
\hline & $100 \sim 109$ & 35 & .066 & \\
\hline & $110 \sim 119$ & 34 & .068 & \\
\hline & $120 \sim 129$ & 11 & .073 & \\
\hline & $130 \sim$ & 9 & .075 & \\
\hline \multicolumn{2}{|c|}{ Mitral stenosis } & 41 & \multicolumn{2}{|c|}{$.081 \pm .009$} \\
\hline
\end{tabular}

* decompensated 15 cases $.077 \pm .010 \mathrm{sec}$.

at the precordial area in the majority of cases. In only 5 cases, it was heard at the base of the heart alone.

No significant difference was noted in different age group of normal individual ( $\mathrm{p}>0.05$ ), and their mean was 0.05 sec. Hypertensive cases occupied the midpoint between normal and mitral stenosis, and its average value was $0.064 \mathrm{sec}$, which was significantly prolonged from the normal value $(\mathrm{p}<0.01)$. As the disease progressed, the value of "Q-1" interval in hypertension became more or less equal to that in mitral stenosis (Fig. 5). This was clearly demonstrated in the cases of hypertension with decompensated 


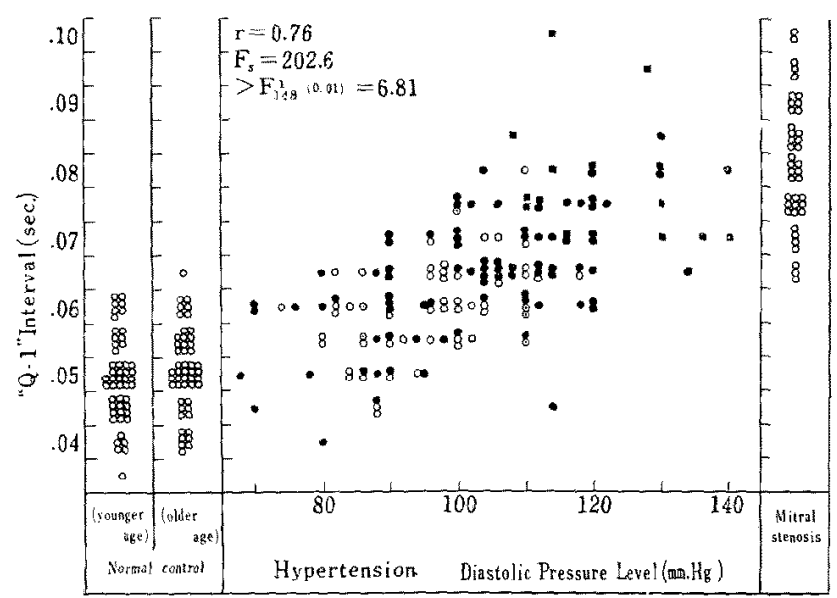

Fig. 5. Relationship of the "Q-1" interval to the diastolic blood pressure level in hypertension.

3) Correlation between the phonocardiographic and electrocardiographic findings:
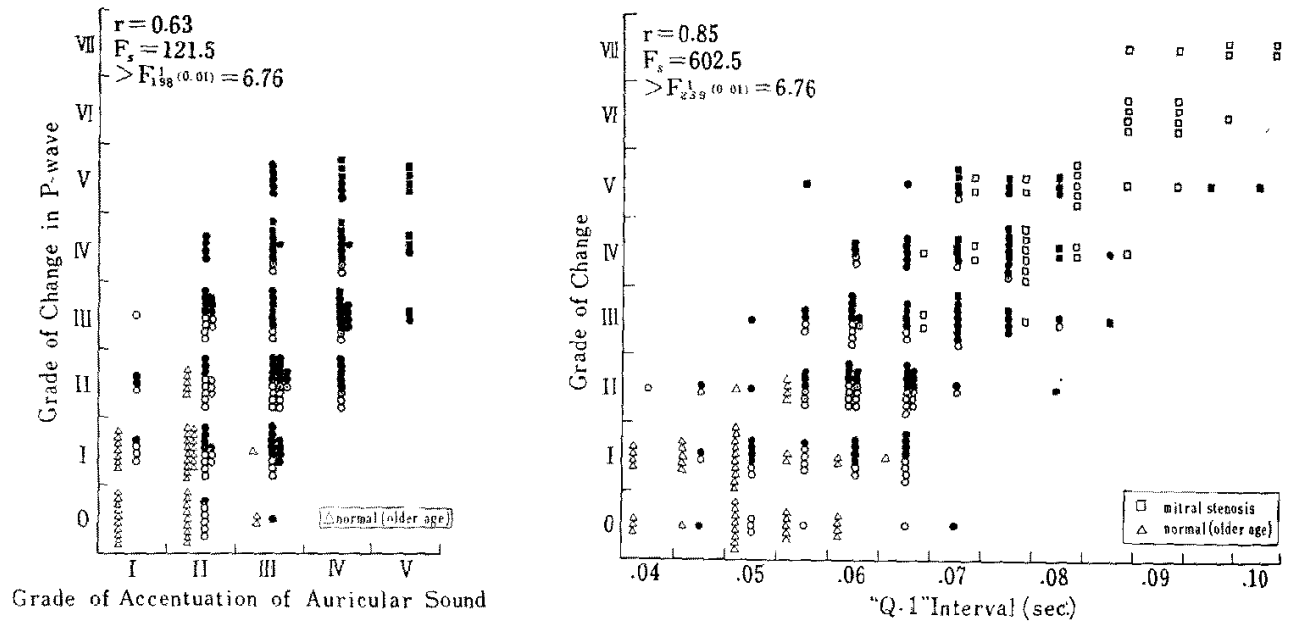

Fig. 6 (Left). Relationship between the grade of accentuation of the atrial sound and the grade of P-wave change.

Fig. 7 (Right). Relationship of the "Q-1" interval to the grade of P-wave change.

heart, in which the average value of this interval $(0.077$ sec.) did not differ from that in mitral stenosis ( $p>0.05$ ).

In 150 cases with hypertension and 50 normal cases, there was a parallelism between the loudness of the atrial sound and the grade of $\mathrm{P}$-wave change (Fig. 6). In a total of 241 cases studied, a significant correlation was revealed between the "Q-1" interval and the grade of the P-wave change (Fig. 7). Their correlation coefficients were 0.54 in hypertension and 0.73 in mitral stenosis. In addition, in 39 cases of mitral stenosis with 
opening snap, there was a significant correlation between the grade of the P-wave change and the "2-OS" interval ${ }^{26)}(\mathrm{r}=-0.61)$, and between the grade of P-wave change and the "( $\mathrm{Q}-1)-(2-\mathrm{OS}) "$ interval ${ }^{26)} \quad(\mathrm{r}=0.75)$. There was also a positive correlation between the " $Q-1$ " interval and the grade of accentuation of the atrial sound (Fig. 8).

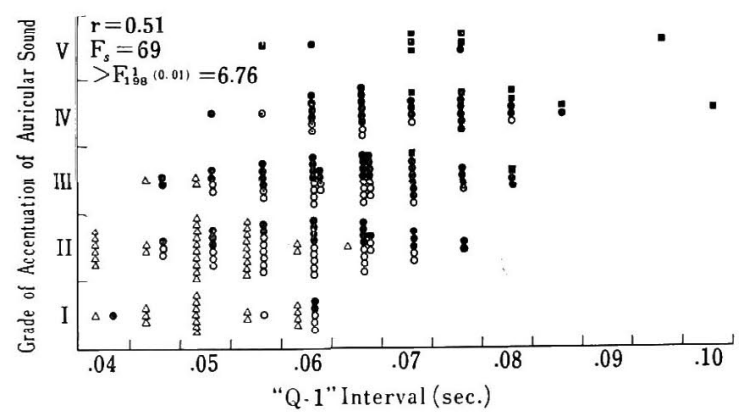

Fig. 8. Relationship of the "Q-1" interval to the grade of accentuation 0 the atrial sound.

4) The changes of phonocardiographic findings in the cold pressor test and follow-up siudy:

All the cases of hypertensive patients who were subjected to the cold pressor test disclosed the elevation of the diastolic blood pressure for an average of $13 \mathrm{~mm} . \mathrm{Hg}$ and showed the accentuated atrial sounds during the test (Fig. $9 \mathrm{a}$ and $\mathrm{b}$ ). In some cases, the accentuation of this sound was 5.5 times greater than the control value, and the average value of accentuation of this sound was 2.5 times greater than that of the control. In 2 cases which developed slight bradycardia during the test also revealed the accentuated atrial sound against

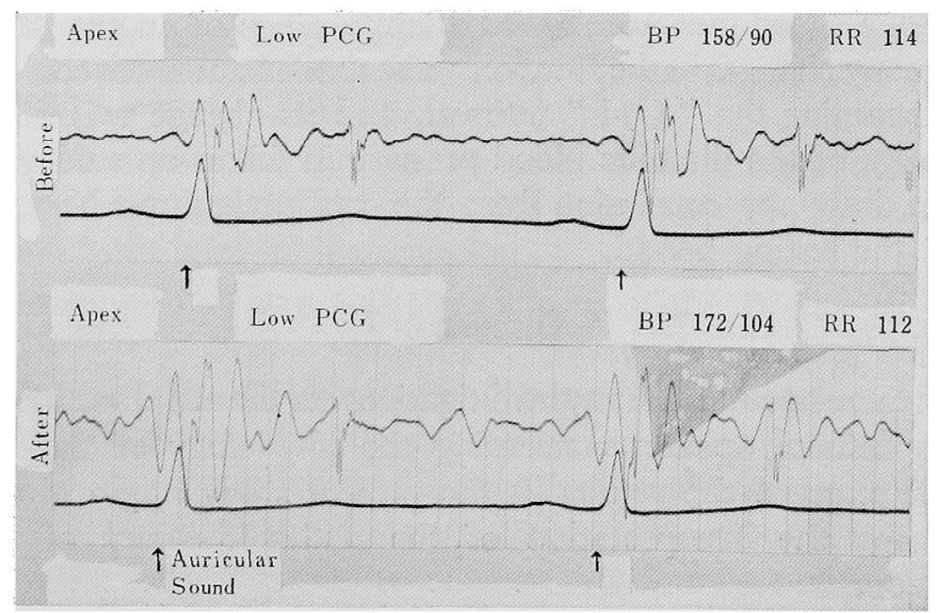

Fig. 9a. Accentuation of the atrial sound caused by the cold pressor test. Marked increase in amplitude of the atrial sound is seen. The other heart sounds also slightly increase in their amplitudes. 


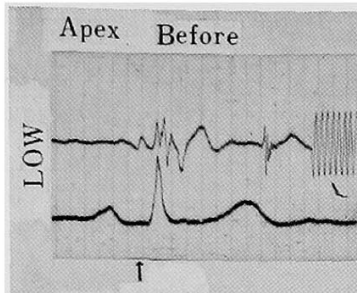

Apex After

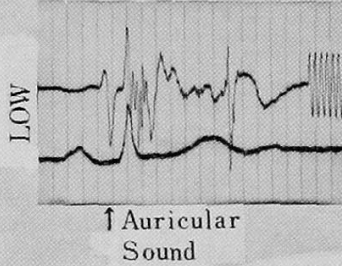

BP $162 / 84 \quad$ RR 100

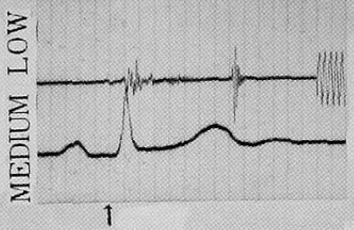

BP $186 / 102$ RR 96

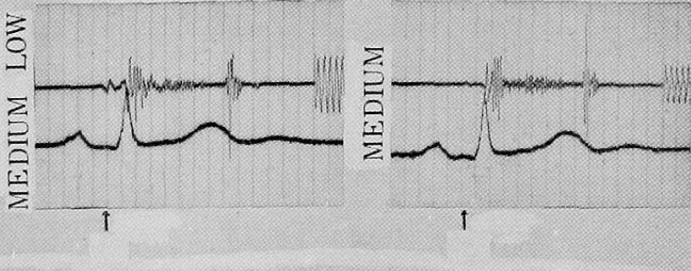

Fig. 9b. Accentuation of the atrial sound caused by the cold pressor test. In addition to the moderate accentuation of the 1 st and 2 nd heart sound, the atrial sound shows the marked accentuation and appears up to Medium PGG durnig the test. The apical systolic murmur become also louder and definitely audible.

our anticipation. This may indicate that the heart rate has no relationship with the loudness of the atrial sound in our study. Both first and second sounds were also accentuated during the cold pressor test, which would make the usual method for the determination of the loudness of the atrial sound unreliable. The meaning of this will be discussed later. The murmurs, both systolic and daistolic, when they were present, were also accentuated during the test. The change in the $\mathrm{P}$-wave appeared in only 2 severe cases as the diastolic blood pressure rose during the test. In 4 follow-up cases with further elevation of diastolic blood pressure, the change in the $\mathrm{P}$-wave and the accentuated atrial sound became more obvious. In contrary to this finding, 2 cases with markedly improved diastolic blood pressure showed disappearance of audible atrial sound as well as many changes in the $\mathrm{P}$-wave. The similar statement could have been obtained concerning the " $Q-1$ " interval, and its change was also reflected with the change of the diastolic blood pressure in follow-up studies, but it was impossible to draw any conclusion from the cold pressor test because of short duration of the testing period.

\section{Discussion}

Though there have been many investigations on the atrial sound in hypertension, ${ }^{3)-10\}}$ no definite opinion concerning the physiology of the atrium was seen. In all respects, the results in the present study might give an useful information for the interpretation of the pathophysiology of the atrium in hypertension.

Although an audibility of the atrial sound is influenced by many factors, ${ }^{11}$ the PCG has a merit for the objective representation of this sound. However, there were some difficulties in differentiating the pathological atrial sound from 
the physiological one. The usual methods for the differentiation between normal and abnormal atrial sound were obtained from the studies with respect to the difference in their frequency ranges ${ }^{18)-16)}$ or the comparison of the amplitudes of the atrial sound with the other heart sounds. ${ }^{17)-20)}$ However, the changes of the first and second heart sound could occur by many factors, therefore, these sounds should not be used for the comparison purpose. ${ }^{211}$ On the other hand, the frequency range of the atrial sound can not be determined accurately by the routine phonocardiographic method. For these reasons, we used to determine the grade of accentuated atrial sound by the finding that up to which PCG this sound could be recorded sufficiently. Of course, it may be possible to use greater amplification and to make the appearance of the atrial sound in higher characteristic PCG in which noraml atrial sound can not be recorded with regular amplification. However, this is impossible in our study because almost every case of hypertension has relatively loud second sound at even apical region. Moreover, as it may be seen in the frequencyresponse characteristics of our amplifier, only loud and/or high frequency atrial sound could be recorded in higher characteristic PCG. Because of these facts, it can be assumed that the atrial sound is accentuated when this sound appears in the higher characteristic PCG. This is well correlated with the diastolic blood pressure in hypertension. In other words, the higher the diastolic blood pressure is, the more the atrial sound is accentuated.

Of course, some technical procedure will influence the intensity of the atrial sound. In order to eliminate this factor and to find that the atrial sound is surely influenced by the change in the diastolic blood pressure, a cold pressor test was carried out. Prior to this test, the microphone was "firmly fixed" to the chest wall and the amplification was regulated at the constant strength. The first recording was made and immediately followed by the second recording during the test. From the results obtained in this test, it was unmistakable that the elevation of the diastolic blood pressure played an important role for the accentuation of the atrial sound. Similar results were also obtained from the follow-up study where the atrial sound appeared in higher characteristic PCG, when there was the increase in the diastolic blood pressure. Then, it may be concluded that the atrial sound is definitely correlated with the diastolic blood pressure level in hypertension.

In follow-up cases, there was the changes of the P-wave in the electrocardiogram besides the louder artial sound due to the increased diastolic blood pressure. However, this change in the P-wave was not evident in an acute experiment in increasing the blood pressure by means in cold pressor test. This would offer an assumption that the change in the $\mathrm{P}$-wave should accompany the sustained elevation of the intra-atrial pressure and some features possibly resulted from the persistent organic changes which might be found in chronic hypertension and not in acute experimentally produced hypertension.

According to Weissler, ${ }^{22)}$ there is a prolongation of the "Q-1" interval in hypertension. As far as the factors which cause this prolongation are con- 
cerned, Kelly ${ }^{23}$ had pointed out two reasons, namely the delay in either electrical or mechanical phenomenon after the onset of the ventricular depolarization. The latter possibility should be applied to explain the prolongation of the "Q-1" interval in our study because the duration of QRS complex in this study is less than $0.10 \mathrm{sec}$. Another similar explanation has been given which explains that the delay of the closure of the atrio-ventricular valves is due to insufficient ventricular contraction as seen in the cases with myocarditis. ${ }^{23}$ In our study, such cases are well ruled out. The remaining cause is seen in the cases with mitral stenosis. In this instance, the prolongation of the "Q-1" interval is mainly due to the delay of the mechanical phenomenon, namely due to the delayed closure of the mitral valve caused by the atrio-ventricular pressure gradient, which is caused by the "mechanical block" due to the stenosis of this valve orifice. This mechanism was conclusively affirmed by Braunwald and his associates" ${ }^{24)}$ and Moscovitz and Wilder. ${ }^{25)}$ Although such a "mechanical block" is not present in hypertension, the possibility may exist that the similar hemodynamic change of the left atrium could be present in both hypertension and mitral stenosis.

The similarity of the circulatory dynamics between the cases with left ventricular failure and mitral stenosis was pointed out by Harvey and his associates, ${ }^{27)}$ and the common denominator of this similarity was the sustained elevation of the left atrial pressure, as reported by Selzer. ${ }^{28)}$ From these observations, it is well understood that the "Q-1 " interval in the hypertensive cases with congestive heart failure in our study does not show a significant difference compared with that scen in mitral stenosis. The P-wave changes in the former should be also similar to that in the latter from the same standpoint of view. A loud atrial sound, which is thought to be corresponding with the presystolic murmur in mitral stenosis, might be also produced in the decompensated hypertensive hearts from the same reasons, since no stenosis of the mitral valve orifice is present in hypertensive cases.

Another discussion should be needed to explain the phonocardiographic changes seen in the hypertensive cases without decompensation. It has been noted that the giant "a " wave of the atrial pressure curve is shown in the presence of systolic overloading of the ventricle as in pulmonary ${ }^{291-31)}$ or

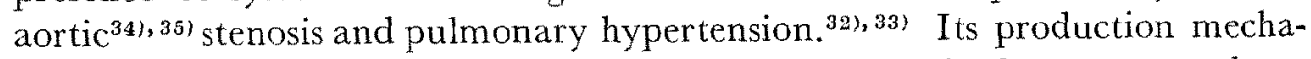
nism, according to these authors, is based on the change in the pressure-volume elasticity relationships of the ventricle, which results in the elevation of its end-diastolic pressure, thus increased resistance to filling of the ventricle reflects itself in the hypertrophy of the atrium with production of the giant " a" wave. The elevation of the end-diastolic pressure in either ventricle appears before the appearance of the clinically recognizable signs of congestive heart failure. ${ }^{331,34)}$ Although the similar finding in the left atrial pressure curve has not been thoroughly investigated in the cases with hypertension, it is likely to assume that there should be the elevated pressure in the left atrium even in the cases of hypertension without decompensation, since 
this condition in the atrium produce both the accentuation of the atrial sound and, when it occurs in the left atrium, the prolongation of the "Q-1" interval. Indeed, the accentuated atrial sound is also seen in the cases with pulmonary stenosis ${ }^{36)}$ and aortic stenosis" in the same fashion as the systemic hypertension(Fig. $10 \mathrm{a}$ and $\mathrm{b}$ ). Moreover, the prolongation of the " $\mathrm{Q}-1$ " interval is frequently observed in the latter ${ }^{37)}$ The $\mathrm{P}$-wave changes in pulmonary stenosis and pulmonary hypertension are well-known, and the P-wave simulating to that seen in mitral stenosis may also be found in severe isolated aortic stenosis (Fig. $10 \mathrm{~b}$ ). Though the prolonged " $\mathrm{Q}-1$ " interval may enhance

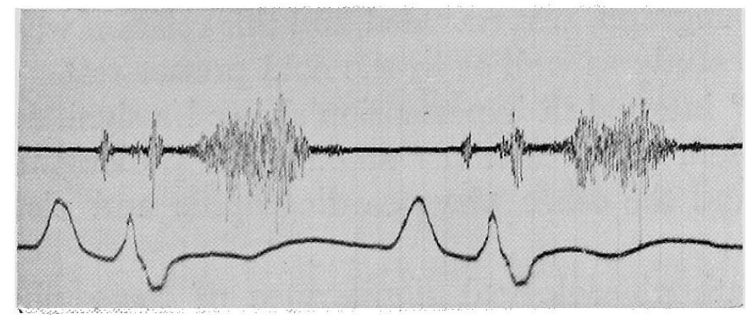

Fig. 10a. Phonocardiogram (Medium High) in a case with pure valvular pulmonary stenosis (Pulmonary area). Loud atrial sound was heard and recognized up to High PGG with its sufficient amplitude. In right heart catheterization, the right ventricular pressure was $150 / 6 \mathrm{~mm} . \mathrm{Hg}$ and the characteristic " a" wave was present (the height $13 \mathrm{~mm} . \mathrm{Hg}$ ) in the right atrial pressure curve.

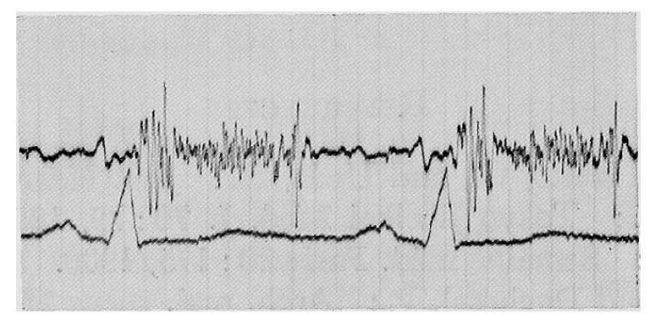

Fig. 10b. Apical phonocardiogram (Medium Low) in a case with pure aortic stenosis without decompensation. An audible atrial sound was recognized up to Medium High PCG. The P-wave is notched. Note the prolongation of the "Q-1" interval $(0.075 \mathrm{sec}$.).

the tendency to accentuate the atrial sound, ${ }^{38)}$ these two phonocardiographic findings seemed to be essentially the same manifestations in the hypertensive hearts in our study.

The elevation of the left atrial pressure caused by the elevated left ventricular end-diastolic pressure might be pathophysiologically unavoidable for hypertension. However, if one interprete this phenomenon as the representation of the preparatory stage of left ventricular failure in hypertension, the electrocardiographic and the phonocardiographic findings seen in this study are considered to indicate the "impending " left heart failure, but not heart failure itself, in hypertensive hearts. Then, our results obtained in this study 
appeared to be of definite value in the assessment of the severity of the hypertension.

\section{Sammary and Conclusions}

Phonocardiographic study was made in order to display the similarity in the left atrial overloading between hypertension and mitral stenosis which is expressed by the $\mathrm{P}$-wave changes as described in the preceeding paper.

(I) A positive correlation among the diastolic blood pressure level and the grade of accentuation of the atrial sound, determined by our multi-filter system phonocardiograph, was revealed and this relation was also ascertained in the follow- up study as well as in the cold pressor test.

(2) " Q-1" interval in hypertension showed a significant prolongation, and there were definite correlations among this prolongation, the diastolic blood pressure, and the other phonocardiographic and electrocardiographic findings.

(3) The pathophysiological significance of these phonocardiographic and electrocardiographic findings in hypertension was discussed. It was concluded that the accentuation of the atrial sound and the prolongation of the "Q-1" interval, combined with the P-wave changes, should be the reflection of the left atrial overloadiug, therefore, these findings might be one of the objective signs indicating the impending left heart failure in hypertensive hearts.

\section{REFERENGES}

1. Sakamoto, T., Kaito, G,, and Ueda, H. : Jap. Heart J, in press.

2. Yoshimura, S.: Tokyo Jikeikai Med. J. 70:79, 1950 (In Japanese).

3. Potain, P. C.: Semaine méd. Paris 20: 175, 1900.

4. Mozer, J. J. and Duchosal, P.: Arch. mal. coeur 23 : 74, 1930.

5. Duchosal, P.: Am. Heart J. $7: 613,1932$.

6. Garvin, G. F.: Am. J. Med. Sci. 205: 814, 1943.

7. Frost, J.: Acta med. scandinav. 134: 153, 1949.

8. Miles, B. E. : Brit. Heart J. 13:327, 1951.

9. Weitzman, D.: Brit. Heart J.: 17: 70, 1955.

10. Sloan, A. W.: Am. Heart J. 55: 715, 1958.

11. Duchosal, P.: Arch. mal. couer $28: 345,1935$.

12. Mannheimer, E.: Acta pediatr. 28: (Suppl. II) 1, 1940.

13. Carlgren, L. E.: Acta pediatr. 33 : (Suppl. VI) 1, 1946.

14. Luisada, A. A.: The Heart Beat, Graphic Methods in the Study of the Cardiac Patient. Paul B. Hoeber, New York, 1953.

15. Schlitter, J. G. and Schölmerich, P.: Cardiologia 26:272, 1955.

16. Luisada, A. A., Richmond, L., and Aravanis, C.: Am. Heart J. 51 : 221, 1956.

17. Luisada, A. A, and Roitman, M.: Arch. Inst. Cardiol. México 17: 345, 1948. cited by Luisada (14). 
18. Sloan, A. W., Campbell, F. W., and Henderson, A. S. : Brit. M. J. 2 : 853, 1952.

19. Engelbertz, P.: Z. Kreislaufforschg. 42:364, 1953.

20. Weissel, W.: Z. Kreislaufforschg. 46:829, 1957.

21. Ueda, H., Sakamoto, T., Kaito, G., Watanabe, H., Uozumi, Z., Nakajima, A., and Kobayashi, T.: Nippon-Rinshō $17: 1366,1959$ (In Japanese).

22. Weissler, A. M., Leonard, J. J., and Warren, J. V. : Circulation 18 : 165, 1958.

23. Kelly, J. J., Jr. : Am. J. Med. 19:862, 1955.

24. Braunwald, E., Moscovitz, H. L., Amram, S. S., Lasser, R. P., Sapin, S. O., Himmelstein, A., Ravitch, M. M., and Gordon, A. I.: Circulation 12: 69, 1955.

25. Moscovitz, H. L. and Wilder, R. J. : Am. Heart J. 53: 741, 1957.

26. Wells, B. G. : Brit. Heart J. $16: 261,1954$.

27. Harvey, R. J., Ferrer, M. I., Samet, P., Bader, R. A., Bader, M. E., Cournand, A., and Richards, D. W., Jr.: Circulation 9: 531, 1955.

28. Selzer, A.: Circulation $20: 243,1959$.

29. Abrahams, D. G. and Wood, P.: Brit. Heart J. 13:519, 1951.

30. Maraist, F., Daley, R., Draper, A., Jr., King, J. T., Fercncz, C., and Bing, R. J.: Bull. Johns Hopkins Hosp. 88: 1, 1951.

31. Engle, M. A. and Taussig, H. B.: Circulation 2: 481, 1950.

32. Dresdale, D. T., Schultz, M., and Michton, R. J.: Am. J. Med. 11 : 686, 1951.

33. McCord, M. C., Komesu, S., and Blount, S. G. : Am. Heart J. 45: 706, 1953.

34. Goldberg, H., Dickens, J. D., Raber, G., and Hayes, E. Jr.: Am. Heart J. 53 : 579, 1957.

35. Goldberg, H., Denton, C., Bender, S., and Uricchio, J. : Dis. Chest 33 : 201, 1958.

36. Benchimol, A. and Dimond, E. G.: Circulation 18:692, 1958 (Abstract).

37. Sakamoto, T.: Jap. Heart J. to be published.

38. Leonard, J. J., Weissler, A. M., and Warren, J. V. : Circulation 17: 1007, 1958. 